\title{
Use of a questionnaire to assess vitamin D status in young adults
}

\author{
Jilaine Bolek-Berquist ${ }^{1, *}+$, Mary E Elliott ${ }^{2,3}$, Ronald E Gangnon ${ }^{4}$, Dessa Gemar ${ }^{5}$, \\ Jean Engelke ${ }^{5}$, Susan J Lawrence ${ }^{1,3}$ and Karen E Hansen ${ }^{1,5}$ \\ 'Department of Medicine, University of Wisconsin School of Medicine \& Public Health, Madison, WI, USA: \\ ${ }^{2}$ Pharmacy Practice Division, University of Wisconsin School of Pharmacy, Madison, WI, USA: ${ }^{3}$ William S. \\ Middleton Veterans Affairs Medical Center, Madison, WI, USA: ${ }^{4}$ Departments of Biostatistics and Medical \\ Informatics and Population Health Sciences, University of Wisconsin, Madison, WI: ${ }^{5}$ Osteoporosis Clinical \\ Center and Research Program, University of Wisconsin-Madison, Madison, WI, USA
}

\section{Submitted 19 October 2007: Accepted 15 July 2008: First published online 27 August 2008}

\begin{abstract}
Objective: We hypothesized that young adults would commonly have vitamin D deficiency and that a questionnaire could help identify subjects with the condition. Design: Between January and May 2004, we administered a questionnaire to a convenience sample of young adults. We measured each participant's serum level of 25-hydroxyvitamin D (25(OH)D) using a chemiluminescent assay and defined deficiency as serum $25(\mathrm{OH}) \mathrm{D}<16 \mathrm{ng} / \mathrm{ml}$.

Setting and subjects: We recruited young adults living in Madison, Wisconsin without pre-existing conditions affecting vitamin D and/or Ca metabolism.

Results: One hundred and eighty-four adults (mean age 24 years, 53\% women, $90 \%$ Caucasian) participated in the study. Nearly three in four adults (71\%) had $25(\mathrm{OH}) \mathrm{D}$ level $<30 \mathrm{ng} / \mathrm{ml}$ and $26 \%$ were vitamin D-deficient. In multivariate analysis, persons reporting a suntan (OR $=0 \cdot 24,95 \%$ CI 0.09, 0.63, $P=0.004)$, tanning booth use $(\mathrm{OR}=0 \cdot 09,95 \% \mathrm{CI} 0 \cdot 02,0 \cdot 43, P=0 \cdot 002)$ and daily ingestion of two or more servings of milk (OR $=0 \cdot 21,95 \%$ CI $0 \cdot 09,0 \cdot 48, P<0 \cdot 001$ ) were less likely to be deficient. These three questions provided a sensitivity and specificity of $79 \%$ and $78 \%$, respectively, for the presence of deficiency.

Conclusions: The questionnaire is moderately useful to identify young adults likely to be vitamin D-deficient. Additional revisions of the questionnaire may improve its ability to predict vitamin D deficiency.
\end{abstract}



Vitamin D deficiency is common in older adults, with recent studies describing deficiency in 18-25\% of adult postmenopausal women ${ }^{(1,2)}$. Vitamin D deficiency results from several factors including inadequate sun exposure, reduced cutaneous vitamin D synthesis, poor nutrition, and certain medications and co-morbid diseases such as anticonvulsants and coeliac sprue ${ }^{(3)}$. The increasing measurement of serum 25-hydroxyvitamin D (25(OH)D) in older people arises from an increasing awareness of the prevalence of hypovitaminosis $\mathrm{D}$ and the role of vitamin $\mathrm{D}$ in both the prevention and management of osteoporosis $^{(4)}$. However, the prevalence of hypovitaminosis D and its impact on health is less certain in adults under 50 years of age.

Healthy young adults may develop vitamin D deficiency for several reasons. First, the vitamin D intake of young adults is often below the recommended intake of

† Correspondence address: c/o Department of Medicine, Section of Rheumatology J5/230-2454, University of Wisconsin Hospital and Clinics, 600 Highland Avenue, Madison, WI 53792, USA.
$200 \mathrm{IU} / \mathrm{d}^{(5,6)}$. Second, young adults today spend less time outside than young adults one decade ago ${ }^{(7-9)}$. Third, the increasing use of sunscreen to reduce skin damage or cancer may decrease or eliminate cutaneous vitamin D synthesis ${ }^{(8,10,11)}$. Finally, many young adults drink carbonated beverages in place of milk, thereby decreasing the intake of both $\mathrm{Ca}$ and vitamin $\mathrm{D}$ and potentially increasing the risk of fracture ${ }^{(12)}$.

Despite mounting evidence that young adults are at risk for vitamin D deficiency, no specific recommendations exist regarding evaluation of their vitamin D status. Such lack of guidelines may result from limited information on either the impact of vitamin D on the development of peak bone mass ${ }^{(13-15)}$ or the long-term safety of increasing serum 25(OH)D levels in young adults. Although epidemiological data suggest that improved vitamin D status may decrease the risk of certain cancers and autoimmune diseases ${ }^{(16-18)}$, a true cause-effect relationship has not been established. Measurement of serum $25(\mathrm{OH}) \mathrm{D}$ is costly, with charges ranging from \$US 45 to \$US 100. A questionnaire to identify persons at 
high or low risk of vitamin D deficiency would be clinically useful, particularly as there is no consensus regarding the indications for measurement of $25(\mathrm{OH}) \mathrm{D}$ in young adults.

Other groups have used questionnaires to detect hypovitaminosis D. One group queried subjects on the use of multivitamins, milk and other foods containing vitamin $\mathrm{D}^{(19)}$ and revealed a positive correlation between serum 25(OH)D levels and multivitamin intake. However, subjects did not record sun exposure. A study in Icelandic women demonstrated an association between higher serum 25(OH)D levels and sun-seeking and dietary habits, but the questionnaire itself was not published ${ }^{(20)}$. Utilizing questions to assess diet and sun exposure, a third group reported associations between serum $25(\mathrm{OH}) \mathrm{D}$ levels and season of measurement, BMI, age, time spent indoors, living in three southern states, vitamin D intake and creatinine. However, the study was limited to elderly subjects ${ }^{(21)}$.

We hypothesized that a simple questionnaire could identify young adults with a high and low likelihood of vitamin $\mathrm{D}$ deficiency. We designed a series of questions to assess use of vitamin D-containing supplements, milk and sun exposure in order to test this hypothesis.

\section{Materials and methods}

Between January and May 2004 we recruited 184 men and women between the ages of 18 and 40 years into the current study. The purposes of the study were twofold. First, we wished to estimate the prevalence of vitamin D deficiency in young adults. Second, we queried whether a questionnaire could identify subjects at high or low risk of vitamin $\mathrm{D}$ deficiency. We excluded individuals with pre-existing conditions affecting vitamin $\mathrm{D}$ and/or Ca metabolism including liver or kidney disease, eating disorders, skin diseases and use of oral corticosteroids, anticonvulsants, insulin or bisphosphonates. We paid volunteers \$US 20 for the single study visit and notified them of vitamin $\mathrm{D}$ test results by mail.

The Human Subjects Committee of the University of Wisconsin approved the study protocol. Participants received verbal and written descriptions of the study, signed the consent form and retained a copy for their records. We recorded the age, gender and self-reported race of each person at the study visit. Each subject completed a questionnaire designed to quantify intake of vitamin $\mathrm{D}$ through diet and sun exposure and to record the presence of conditions or medications known to affect vitamin D stores (Table 1).

To measure $25(\mathrm{OH}) \mathrm{D}$, we collected blood from nonfasting participants ${ }^{(22)}$ and transported samples, without exposure to light, to a central laboratory at the University of Wisconsin. Samples were stored at $-70^{\circ} \mathrm{C}$ until analysis. Subsequently, we measured serum $25(\mathrm{OH}) \mathrm{D}$ using a
Table 1 The Vitamin D Questionnaire

1. Have you received a suntan in the past 12 months? Yes or No

2. Do you use sunscreen? Yes or No

3. On average, how much sun exposure have you had in the past week?

less than 5 minutes per day, 5-15 minutes per day, 15-30 minutes per day, more than 30 minutes per day

4. Have you used a tanning booth in the past year? Yes or No

5. How many servings of milk do you get daily?

6. Do you take multivitamins? Yes or No If yes, how many multivitamin tablets do you take daily?

7. Do you take vitamin $D$ supplements or calcium with vitamin $D$ ? Yes or No

If so, how many IU per day?

8. Do you take cod-liver oil or omega-3 fatty acids (fish oil)? Yes or No

9. What is your ethnic background?

10. Have you been diagnosed with Crohn's disease, ulcerative colitis or coeliac sprue? Yes or No

11. Have you had diarrhoea in the past two weeks? Yes or No

Liaison chemiluminescence assay (DiaSorin Inc., Stillwater, MN, USA). The chemiluminescence assay is an accurate, rapid and precise method for vitamin D measurement, correlating well with traditional radioimmunoassay but overestimating levels by $3.9 \mathrm{ng} / \mathrm{ml}$ compared with $\operatorname{HPLC}^{(23-25)}$. In a study of 329 clinical samples, the intra- and inter-assay $\mathrm{CV}$ for this assay were $8-13 \%$ and $8-15 \%$, respectively ${ }^{(25)}$.

The precise cut-off points used to define vitamin $\mathrm{D}$ adequacy, insufficiency and deficiency vary, depending on the assay utilized and the investigator. However, many experts use a $25(\mathrm{OH}) \mathrm{D}$ level $<30 \mathrm{ng} / \mathrm{ml}(75 \mathrm{nmol} / \mathrm{l})$ to define vitamin $D$ insufficiency and a level $<16 \mathrm{ng} / \mathrm{ml}$ $(40 \mathrm{nmol} / \mathrm{l})$ to define deficiency ${ }^{(3)}$. For the purposes of the present study, we used serum $25(\mathrm{OH}) \mathrm{D}<16 \mathrm{ng} / \mathrm{ml}$ to categorize individuals as vitamin D-deficient.

\section{Statistical analysis}

We summarized data as the mean and standard deviation for continuous variables and as frequencies and percentages for categorical variables. We compared vitamin D-sufficient and -deficient subjects using the Wilcoxon rank-sum test for continuous variables and the $\chi^{2}$ or Fisher's exact test for categorical variables. We used univariate and multivariate logistic regression models to evaluate the effects of questionnaire responses on odds ratios for vitamin D deficiency. We controlled for age and gender in multivariate analysis. This is because age and gender are significantly associated with the likelihood of vitamin $\mathrm{D}$ deficiency and both likely affect nutritional and sun-seeking habits. We assessed the sensitivity and specificity of combinations of questions in their ability to identify subjects with vitamin D deficiency. We completed analyses using SAS version 9.1 (SAS Institute, Cary, NC, USA) and $\mathrm{R}$ version $2 \cdot 4 \cdot 0$ (The $\mathrm{R}$ Project for Statistical Computing, http://www.r-project.org) statistical software packages. 


\section{Results}

We recruited 184 subjects for the study. Two-thirds of subjects ( $n$ 124) participated during winter and 33\% ( $n$ 60) during the spring. Subjects' mean (SD) age was 24 (4) years while the median age and range were 22.4 years and 18 to 40 years, respectively. Over half of the subjects $(53 \%, n 98)$ were female and 90\% ( $n$ 165) were Caucasian (Table 2 ). Mean (SD) serum 25(OH)D levels were 25 (11) ng/ml (range 4 to $52 \mathrm{ng} / \mathrm{ml})$. Nearly three in four subjects $(71 \%, n 130)$ had serum $25(\mathrm{OH}) \mathrm{D}$ level $<30 \mathrm{ng} / \mathrm{ml}$ and one in four (26\%, $n$ 48) subjects were vitamin D-deficient.

Table 2 summarizes the entire group's answers to the questionnaire. Participants reported drinking an average of $1.9(1.5)$ servings of milk daily. Nearly half of the subjects ( $46 \%, n$ 84) ingested a daily multivitamin but only $6 \%(n 11)$ took an additional vitamin D supplement and $3 \%(n 5)$ reported daily use of cod-liver oil. Subjects' mean vitamin D intake through milk was 188 (148) IU and through supplements was 77 (238) IU daily. Eighty-five per cent of responders ( $n$ 157) reported sun tanning, $88 \%$ ( $n$ 161) reported sunscreen use, 29\% ( $n 53)$ reported over $30 \mathrm{~min}$ of sun exposure daily and 35\% ( $n$ 64) reported tanning booth use in the past year.

Several characteristics identified subjects more likely to have vitamin D deficiency (Table 3). Individuals with vitamin $\mathrm{D}$ deficiency reported lower milk intake $(1 \cdot 2 \mathrm{v}$. $2 \cdot 1$ servings $/ \mathrm{d}, P<0 \cdot 001)$. Subjects with deficiency were less likely to report a suntan $(71 \% v .90 \%, P<0 \cdot 001)$ or use a tanning booth ( $4 \% v .46 \%, P<0 \cdot 001)$. Individuals with vitamin $\mathrm{D}$ deficiency were slightly older (mean age 26.5 years $v .22 .9$ years, $P<0.001$ ) and more often male (65\% v. 40\%, $P=0 \cdot 005$ ). Finally, non-Caucasian individuals were more likely to be vitamin D-deficient than Caucasians (deficiency in $67 \%$ of non-Caucasians v. $23 \%$ of Caucasians, $P<0 \cdot 001$ ).

We performed univariate and multivariate logistic regression analyses to determine the odds ratio for vitamin D deficiency based on individual items in the questionnaire (Table 4). In univariate results, older age and male gender conferred a greater odds ratio for vitamin D deficiency. We controlled for age and gender in multivariate analyses for two reasons. First, age and gender may themselves be associated with differing nutritional and sun-seeking habits. Second, other studies disagree on whether older age and male gender are risk factors for vitamin D deficiency ${ }^{(21,26,27)}$. Results from adjusted and unadjusted analyses were similar. In multivariate analyses, adults under the median age of 22.4 years $(O R=$ $0 \cdot 25,95 \%$ CI $0 \cdot 12,0 \cdot 53, P<0 \cdot 001)$, those reporting a suntan $(\mathrm{OR}=0 \cdot 24,95 \% \mathrm{CI} 0 \cdot 09,0 \cdot 63, P=0 \cdot 004)$, tanning booth use (OR $=0.09,95 \% \mathrm{CI} 0 \cdot 02,0 \cdot 43, P=0 \cdot 002)$ and daily ingestion of two or more servings of milk (OR = $0 \cdot 21,95 \%$ CI $0 \cdot 09,0 \cdot 48, P<0 \cdot 001$ ) were less likely to be deficient. In contrast, individuals more likely to be deficient were non-Caucasians $(\mathrm{OR}=5 \cdot 50,95 \%$ CI $1 \cdot 35$,
Table 2 Participants' demographics and answers to the vitamin $D$ questionnaire: young adults ( $n$ 184) living in Madison, Wisconsin, January-May 2004

\begin{tabular}{|c|c|c|}
\hline Characteristic & Mean or $n$ & SD or $\%$ \\
\hline Serum 25(OH)D (ng/ml)* & 25 & 11 \\
\hline Vitamin $\mathrm{D}$ deficiency & 48 & 26 \\
\hline 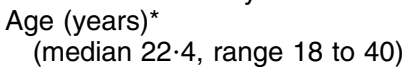 & 24 & 4 \\
\hline Female gender & 98 & 53 \\
\hline \multicolumn{3}{|l|}{ Ethnicity } \\
\hline Asian & 11 & 6 \\
\hline African American & 1 & $<1$ \\
\hline Caucasian & 165 & 90 \\
\hline Hispanic & 1 & $<1$ \\
\hline Native American & 2 & 1 \\
\hline Other/no answer & 4 & 2 \\
\hline \multicolumn{3}{|l|}{ Season of $25(\mathrm{OH})$ D collection } \\
\hline Spring & 60 & 33 \\
\hline Winter & 124 & 67 \\
\hline \multicolumn{3}{|l|}{ Suntan } \\
\hline Yes & 157 & 85 \\
\hline No & 25 & 14 \\
\hline No answer & 2 & 1 \\
\hline \multicolumn{3}{|l|}{ Sunscreen } \\
\hline Yes & 161 & 88 \\
\hline No & 21 & 11 \\
\hline No answer & 2 & 1 \\
\hline \multicolumn{3}{|l|}{ Sun exposure } \\
\hline$<5 \mathrm{~min} / \mathrm{d}$ & 16 & 9 \\
\hline $5-15 \mathrm{~min} / \mathrm{d}$ & 38 & 21 \\
\hline $15-30 \mathrm{~min} / \mathrm{d}$ & 75 & 40 \\
\hline$>30 \mathrm{~min} / \mathrm{d}$ & 53 & 29 \\
\hline No answer & 2 & 1 \\
\hline \multicolumn{3}{|l|}{ Tanning booth } \\
\hline Yes & 64 & 35 \\
\hline No & 118 & 64 \\
\hline No answer & 2 & 1 \\
\hline Milk (servings/d)* & $1 \cdot 9$ & 1.5 \\
\hline \multicolumn{3}{|l|}{ Multivitamin use } \\
\hline Yes & 84 & 46 \\
\hline No & 98 & 53 \\
\hline No answer & 2 & 1 \\
\hline \multicolumn{3}{|l|}{ Vitamin D supplement use } \\
\hline Yes & 11 & 6 \\
\hline No & 170 & 92 \\
\hline No answer & 3 & 2 \\
\hline \multicolumn{3}{|l|}{ Cod-liver oil or fish oil use } \\
\hline Yes & 5 & 3 \\
\hline No & 175 & 95 \\
\hline No answer & 4 & 2 \\
\hline Total vitamin D intake $(\mathrm{IU} / \mathrm{d})^{\star}$ & 265 & 258 \\
\hline From milk* & 188 & 148 \\
\hline From supplements* & 77 & 238 \\
\hline \multicolumn{3}{|l|}{ Chronic intestinal disorder } \\
\hline Yes & 1 & 1 \\
\hline No & 179 & 97 \\
\hline \multirow{2}{*}{\multicolumn{3}{|c|}{ Recent diarrhoea }} \\
\hline & & \\
\hline Yes & 8 & 4 \\
\hline No & 171 & 93 \\
\hline No answer & 5 & 3 \\
\hline
\end{tabular}

25(OH)D, 25-hydroxyvitamin D.

Data are shown as mean values with their standard deviation (indicated by *) or as numbers and percentage.

$22 \cdot 41, P=0 \cdot 02)$ and men $(\mathrm{OR}=3 \cdot 44,95 \%$ CI $1 \cdot 60,7 \cdot 37$, $P=0 \cdot 002)$. We performed additional univariate and multivariate analyses without non-Caucasian subjects ( $n$ 19); these analyses showed virtually identical findings (Table 5). 
Table 3 Characteristics of subjects with and without vitamin D deficiency: young adults $(n$ 184) living in Madison, Wisconsin, January-May 2004

\begin{tabular}{|c|c|c|c|c|c|}
\hline \multirow[b]{2}{*}{ Characteristic } & \multicolumn{2}{|c|}{ Vitamin D deficient $(n 48)$} & \multicolumn{2}{|c|}{ Vitamin D sufficient ( $n$ 136) } & \multirow[b]{2}{*}{$P$ value } \\
\hline & Mean or $n$ & SD or $\%$ & Mean or $n$ & SD or $\%$ & \\
\hline Serum $25(\mathrm{OH}) \mathrm{D}(\mathrm{ng} / \mathrm{ml})^{*}$ & 12 & 3 & 29 & 10 & $<0.0001$ \\
\hline Age (years) ${ }^{*}$ & $26 \cdot 5$ & $5 \cdot 6$ & $22 \cdot 9$ & $3 \cdot 1$ & $<0.001$ \\
\hline Male gender & 31 & 65 & 55 & 40 & 0.005 \\
\hline \multicolumn{6}{|l|}{ Ethnicity } \\
\hline Caucasian & 38 & 81 & 127 & 97 & 0.001 \\
\hline Non-Caucasian & 9 & 19 & 4 & 3 & \\
\hline \multicolumn{6}{|l|}{ Season of $25(\mathrm{OH})$ D collection } \\
\hline Spring & 18 & 38 & 42 & 31 & $0 \cdot 44$ \\
\hline Winter & 30 & 63 & 94 & 69 & 0.40 \\
\hline Suntan & 34 & 71 & 123 & 90 & $<0.001$ \\
\hline Sunscreen & 40 & 83 & 121 & 90 & $0 \cdot 20$ \\
\hline \multicolumn{6}{|l|}{ Sun exposure } \\
\hline$<5 \mathrm{~min} / \mathrm{d}$ & 5 & 10 & 11 & 8 & 0.99 \\
\hline $5-15 \mathrm{~min} / \mathrm{d}$ & 11 & 23 & 27 & 20 & 0.96 \\
\hline $15-30 \mathrm{~min} / \mathrm{d}$ & 18 & 38 & 57 & 42 & 0.96 \\
\hline$>30 \mathrm{~min} / \mathrm{d}$ & 14 & 29 & 39 & 29 & 0.96 \\
\hline Tanning booth & 2 & 4 & 62 & 46 & $<0.001$ \\
\hline Milk (servings/d)* & $1 \cdot 2$ & $1 \cdot 6$ & $2 \cdot 1$ & 1.5 & $<0.001$ \\
\hline Multivitamin use & 17 & 35 & 67 & 50 & 0.08 \\
\hline Vitamin D supplement use & 3 & 6 & 8 & 6 & 0.95 \\
\hline Cod-liver oil or fish oil use & 3 & 6 & 2 & 2 & 0.09 \\
\hline Use of any tablet with vitamin D & 19 & 40 & 69 & 51 & $0 \cdot 19$ \\
\hline Total vitamin $D$ intake $(\mathrm{IU} / \mathrm{d})^{\star}$ & 242 & 333 & 273 & 226 & 0.47 \\
\hline From milk* & 121 & 103 & 211 & 154 & $<0.001$ \\
\hline From supplements* & 121 & 342 & 62 & 187 & $0 \cdot 14$ \\
\hline Chronic intestinal disorder & 0 & & 1 & 1 & 0.54 \\
\hline Recent diarrhoea & 0 & & 8 & 6 & 0.08 \\
\hline
\end{tabular}

25(OH)D, 25-hydroxyvitamin D.

Data are shown as mean values with their standard deviation (indicated by ${ }^{\star}$ ) or as numbers and percentage.

Table 4 Univariate and multivariate odds ratios for vitamin D deficiency: young adults $(n$ 184) living in Madison, Wisconsin, January-May 2004

\begin{tabular}{|c|c|c|c|c|c|c|}
\hline & \multicolumn{3}{|c|}{ Univariate model } & \multicolumn{3}{|c|}{ Multivariate model $^{*}$} \\
\hline & OR & $95 \% \mathrm{Cl}$ & $P$ value & OR & $95 \% \mathrm{Cl}$ & $P$ value \\
\hline \multicolumn{7}{|l|}{ Demographic variables } \\
\hline Age $<22 \cdot 4$ years & $0 \cdot 30$ & $0.14,0.63$ & $<0.001$ & 0.25 & $0.12,0.53$ & $<0.001$ \\
\hline Male gender & $2 \cdot 67$ & $1 \cdot 29,5 \cdot 69$ & 0.004 & $3 \cdot 44$ & $1 \cdot 60,7 \cdot 37$ & 0.002 \\
\hline Non-Caucasian & $7 \cdot 41$ & $1 \cdot 94,34 \cdot 82$ & $<0.001$ & $5 \cdot 50$ & $1 \cdot 35,22 \cdot 41$ & 0.02 \\
\hline \multicolumn{7}{|l|}{ Sun-seeking habits } \\
\hline Winter season & $0 \cdot 75$ & $0 \cdot 36,1 \cdot 59$ & 0.47 & $1 \cdot 22$ & $0.53,2.79$ & 0.64 \\
\hline Suntan & $0 \cdot 22$ & $0.08,0.57$ & $<0.001$ & $0 \cdot 24$ & $0.09,0.63$ & 0.004 \\
\hline Sunscreen & 0.54 & $0 \cdot 19,1 \cdot 61$ & $0 \cdot 20$ & $0 \cdot 72$ & $0 \cdot 24,2 \cdot 16$ & 0.56 \\
\hline \multicolumn{7}{|l|}{ Sun exposure } \\
\hline$<5 \mathrm{~min} / \mathrm{d}$ & $1 \cdot 30$ & $0 \cdot 33,4 \cdot 34$ & $0 \cdot 77$ & $0 \cdot 85$ & $0.24,3.07$ & $0 \cdot 81$ \\
\hline $5-15 \mathrm{~min} / \mathrm{d}$ & $1 \cdot 18$ & $0 \cdot 48,2 \cdot 75$ & 0.68 & 1.54 & $0.62,3 \cdot 83$ & 0.35 \\
\hline $15-30 \mathrm{~min} / \mathrm{d}$ & $0 \cdot 81$ & $0.39,1.67$ & 0.61 & 0.96 & $0.45,2 \cdot 06$ & 0.92 \\
\hline$>30 \mathrm{~min} / \mathrm{d}$ & $1 \cdot 00$ & $0 \cdot 45,2 \cdot 17$ & $1 \cdot 0$ & $0 \cdot 84$ & $0.36,1.94$ & 0.68 \\
\hline Tanning booth & 0.05 & $0.006,0.21$ & $<0.001$ & 0.09 & $0.02,0.43$ & 0.002 \\
\hline \multicolumn{7}{|l|}{ Dietary habits } \\
\hline Milk $(2+$ servings/d) & 0.28 & $0.13,0.59$ & $<0.001$ & $0 \cdot 21$ & $0.09,0.48$ & $<0.001$ \\
\hline Multivitamin use & 0.55 & $0 \cdot 26,1 \cdot 14$ & 0.09 & 0.51 & $0 \cdot 23,1 \cdot 11$ & 0.09 \\
\hline Vitamin D supplement use & 1.04 & $0 \cdot 17,4 \cdot 58$ & $1 \cdot 0$ & $1 \cdot 39$ & $0 \cdot 29,6 \cdot 72$ & $0 \cdot 68$ \\
\hline Fish oil use & $4 \cdot 29$ & $0 \cdot 48,52 \cdot 9$ & $0 \cdot 12$ & $3 \cdot 31$ & $0 \cdot 42,25 \cdot 90$ & $0 \cdot 26$ \\
\hline Use of any tablet with vitamin D & $0 \cdot 62$ & $0 \cdot 30,1 \cdot 27$ & $0 \cdot 18$ & 0.51 & $0 \cdot 23,1 \cdot 12$ & 0.09 \\
\hline \multicolumn{7}{|l|}{ Intestinal conditions } \\
\hline Chronic intestinal disorder & 0 & 0,107 & 0.55 & 0 & 0 , infinity & 0.99 \\
\hline Recent diarrhoea & 0 & $0,1 \cdot 62$ & $0 \cdot 11$ & 0 & 0 , infinity & 0.99 \\
\hline
\end{tabular}

*Multivariate analyses are adjusted for age and gender. 
Table 5 Univariate and multivariate odds ratios for vitamin D deficiency in Caucasian subjects: young adults $(n$ 165) living in Madison, Wisconsin, January-May 2004

\begin{tabular}{|c|c|c|c|c|c|c|}
\hline & \multicolumn{3}{|c|}{ Univariate model } & \multicolumn{3}{|c|}{ Multivariate model ${ }^{*}$} \\
\hline & OR & $95 \% \mathrm{Cl}$ & $P$ value & OR & $95 \% \mathrm{Cl}$ & $P$ value \\
\hline \multicolumn{7}{|l|}{ Demographic variables } \\
\hline Age $<22 \cdot 4$ years & 0.33 & $0 \cdot 14,0 \cdot 76$ & 0.005 & 0.28 & $0.13,0.63$ & 0.002 \\
\hline Male gender & $2 \cdot 67$ & $1 \cdot 19,6 \cdot 24$ & $0 \cdot 01$ & $3 \cdot 19$ & $1 \cdot 44,7 \cdot 05$ & 0.004 \\
\hline \multicolumn{7}{|l|}{ Sun-seeking habits } \\
\hline Winter season & 0.88 & $0.39,2.09$ & 0.84 & $4 \cdot 18$ & $0 \cdot 67,4 \cdot 18$ & $0 \cdot 27$ \\
\hline Suntan & 0.25 & $0.08,0.78$ & 0.007 & 0.21 & $0.07,0.64$ & 0.006 \\
\hline Sunscreen & 0.73 & $0 \cdot 19,3 \cdot 38$ & 0.74 & 0.72 & $0.20,2 \cdot 66$ & 0.62 \\
\hline \multicolumn{7}{|l|}{ Sun exposure } \\
\hline$<5 \mathrm{~min} / \mathrm{d}$ & $1 \cdot 24$ & $0.27,4.53$ & $0 \cdot 75$ & 0.63 & $0 \cdot 15,2 \cdot 64$ & 0.53 \\
\hline $5-15 \mathrm{~min} / \mathrm{d}$ & 0.88 & $0 \cdot 29,2 \cdot 34$ & $1 \cdot 0$ & $1 \cdot 43$ & $0.52,3.94$ & 0.49 \\
\hline $15-30 \mathrm{~min} / \mathrm{d}$ & 0.82 & $0.35,1.82$ & $0 \cdot 71$ & 0.87 & $0.38,2 \cdot 00$ & 0.75 \\
\hline$>30 \mathrm{~min} / \mathrm{d}$ & $1 \cdot 26$ & $0.53,2.90$ & 0.55 & $1 \cdot 08$ & $0 \cdot 45,2 \cdot 61$ & $0 \cdot 85$ \\
\hline Tanning booth & 0.06 & $0.007,0.27$ & $<0.001$ & $0 \cdot 11$ & $0.02,0.52$ & 0.005 \\
\hline \multicolumn{7}{|l|}{ Dietary habits } \\
\hline Milk $(2+$ servings/d) & $0 \cdot 30$ & $0.13,0.69$ & 0.003 & 0.25 & $0.10,0.59$ & 0.002 \\
\hline Multivitamin use & 0.69 & $0.30,1.52$ & 0.36 & 0.61 & $0 \cdot 27,1 \cdot 41$ & $0 \cdot 25$ \\
\hline Vitamin D supplement use & $1 \cdot 72$ & $0.27,8.57$ & 0.43 & $2 \cdot 29$ & $0 \cdot 42,12 \cdot 4$ & 0.34 \\
\hline Fish oil use & $5 \cdot 29$ & $0 \cdot 58,65 \cdot 7$ & 0.08 & $3 \cdot 76$ & $0 \cdot 47,29 \cdot 8$ & $0 \cdot 21$ \\
\hline \multicolumn{7}{|l|}{ Intestinal conditions } \\
\hline Chronic intestinal disorder & 0 & 0,130 & $1 \cdot 0$ & 0 & 0 , infinity & 0.99 \\
\hline Recent diarrhoea & 0 & $0,1.99$ & 0.20 & 0 & 0 , infinity & 0.99 \\
\hline
\end{tabular}

${ }^{*}$ Multivariate analyses are adjusted for age and gender.

Table 6 Sensitivity and specificity of three questions ${ }^{*}$ for vitamin $D$ deficiency: young adults ( $n$ 184) living in Madison, Wisconsin, January-May 2004

\begin{tabular}{lrc}
\hline & $\begin{array}{c}\text { Sensitivity } \\
(\%)\end{array}$ & $\begin{array}{c}\text { Specificity } \\
(\%)\end{array}$ \\
\hline Three questions answered 'no' & 19 & 98 \\
Two or more questions answered 'no' & 79 & 78 \\
One or more questions answered 'no' & 96 & 25 \\
Any questions answered 'no' & 100 & 0 \\
\hline
\end{tabular}

${ }^{*}$ Questions: (i) Have you received a suntan in the past 12 months? Yes or No; (ii) Have you used a tanning booth in the past year? Yes or No; (iii) How many servings of milk do you get daily? Two or more servings $=$ Yes.

We evaluated the sensitivity and specificity of the questionnaire as a screening test for vitamin D deficiency (Table 6). Three self-reported habits (suntan, tanning booth use and drinking two or more servings of milk daily) discriminated between young adults with and without vitamin D deficiency. Using a threshold of two out of three negative responses for these habits, we obtained a sensitivity of $79 \%$ and a specificity of $78 \%$ for identifying persons with vitamin $\mathrm{D}$ deficiency. We obtained a similar sensitivity (76\%) and specificity (79\%) when excluding non-Caucasian subjects (Table 7).

\section{Discussion}

We hypothesized that, like other age groups, young adults would commonly have vitamin D deficiency. In this study of 184 healthy young subjects, $71 \%$ had serum
Table 7 Sensitivity and specificity of three questions* for vitamin D deficiency in Caucasian subjects: young adults ( $n$ 165) living in Madison, Wisconsin, January-May 2004

\begin{tabular}{lrr}
\hline & $\begin{array}{r}\text { Sensitivity } \\
(\%)\end{array}$ & $\begin{array}{r}\text { Specificity } \\
(\%)\end{array}$ \\
\hline Three questions answered 'no' & 13 & 98 \\
Two or more questions answered 'no' & 76 & 79 \\
One or more question answered 'no' & 95 & 25 \\
Any questions answered 'no' & 100 & 0 \\
\hline
\end{tabular}

*Questions: (i) Have you received a suntan in the past 12 months? Yes or No; (ii) Have you used a tanning booth in the past year? Yes or No; (iii) How many servings of milk do you get daily? Two or more servings $=$ Yes.

$25(\mathrm{OH}) \mathrm{D}$ level $<30 \mathrm{ng} / \mathrm{ml}$ and $26 \%$ were clearly vitamin D-deficient, with 25(OH)D level below $16 \mathrm{ng} / \mathrm{ml}$. People with vitamin $\mathrm{D}$ deficiency may develop osteomalacia, a disease characterized by unmineralized osteoid leading to bone pain and skeletal fragility. Higher vitamin D levels are associated with increased bone mass ${ }^{(13-15,28)}$. Indeed, studies suggest that preventing vitamin D deficiency may optimize Ca homeostasis and facilitate peak bone mass in young adults ${ }^{(13,14)}$. The high prevalence of deficiency in young adults highlights the need for further research to identify the precise vitamin D level needed to optimize musculoskeletal health. Such knowledge will facilitate patient education and public policy, with the goal of achieving vitamin $\mathrm{D}$ adequacy.

We hypothesized that a questionnaire could identify young people at high and low risk of vitamin D deficiency. A single question did not reliably distinguish between these groups. However, those subjects who 
received a suntan, used a tanning booth or drank at least two servings of milk daily were significantly less likely to be deficient than subjects not reporting these habits. In combination, these three items were useful in differentiating between those with and without vitamin D deficiency. For subjects responding in the negative to any two of these three questions, we obtained a sensitivity of $79 \%$ and specificity of $78 \%$ for predicting vitamin D deficiency. Although the questionnaire needs further revision to improve its performance, it appears that three questions may help clinicians decide whether to pursue laboratory testing for vitamin D deficiency.

It is not surprising that sun exposure and milk ingestion may protect against vitamin D deficiency. Vitamin D fortification of milk is required in the USA, based on research carried out decades ago at the University of Wisconsin. An 8 ounce glass of milk contains $\sim 100 \mathrm{IU}$ of vitamin D. Likewise, cutaneous sun exposure increases $25(\mathrm{OH}) \mathrm{D}$ levels, unless sunscreen with a sun protection factor $>15$ is used $^{(29)}$. Additionally, many tanning beds emit UV-B light, which increases vitamin D synthesis ${ }^{(20,30,31)}$. Although suninduced summer increments in serum 25(OH)D gradually decline over the winter, women with low vitamin D intake but high summer sun exposure may maintain higher serum $25(\mathrm{OH}) \mathrm{D}$ levels in the winter as well ${ }^{(32)}$. While the explanation for this observation is unknown, summer sun exposure was associated in one study with improved vitamin D status year-round ${ }^{(32)}$.

Few studies have used questionnaires to predict low serum $25(\mathrm{OH}) \mathrm{D}$ levels. In a study by Tangpricha et al., serum 25(OH)D levels were higher in subjects taking multivitamins, but not higher in milk drinkers ${ }^{(19)}$. The authors analysed these associations between habits and serum $25(\mathrm{OH}) \mathrm{D}$ levels ${ }^{(19)}$, rather than stratifying subjects as sufficient or deficient as we did. Our vitamin D-deficient subjects were less likely to take multivitamins compared with sufficient subjects $(35 \% v .50 \%, P=0 \cdot 08)$ but further analyses showed no effect of multivitamin use on the odds of vitamin D deficiency. Milk consumption in Tangpricha's study was lower (mean 1.6 (SD 1) servings/d) ${ }^{(19)}$ than that reported by our subjects, which might explain why there was no difference in vitamin $\mathrm{D}$ levels between their subjects who drank and did not drink milk. In a questionnairebased Icelandic study, older women consuming fish oil or multivitamins had higher serum 25(OH)D levels $(P<0 \cdot 01)$ than younger women who did not report these habits ${ }^{(20)}$. Women whose used tanning beds $(P=0 \cdot 06)$ or travelled to warmer climates $(P<0 \cdot 01)$ also had higher $25(\mathrm{OH}) \mathrm{D}$ levels ${ }^{(20)}$. A third study demonstrated associations between serum 25(OH)D and season, BMI, age, time spent indoors, living in southern states, vitamin D intake and creatinine ${ }^{(21)}$. Taken as a whole, many of these findings are very similar to ours and suggest that questions about sun exposure and supplemental and dietary vitamin D intake may be universally useful to identify individuals at risk for vitamin D deficiency.
Based on larger cross-sectional studies ${ }^{(26)}$, we do not believe that questions about age will prove useful to exclude or suspect vitamin D deficiency. Indeed, ours is not the first study to report vitamin $\mathrm{D}$ inadequacy in young adults. In Iceland, younger women had lower vitamin D levels than older women ${ }^{(20)}$. Nearly half of adolescent girls in Maine had hypovitaminosis D at least once during a three-year observation ${ }^{(33)}$. Two-thirds of internal medicine residents had 25(OH)D levels below $20 \mathrm{ng} / \mathrm{ml}$ during spring months in Oregon ${ }^{(34)}$. Together these studies indicate that hypovitaminosis D is common in young adults.

One unexpected finding in the present study was that men were more likely than women to be vitamin D-deficient. In large epidemiology studies, men typically have higher serum $25(\mathrm{OH}) \mathrm{D}$ levels than women ${ }^{(21,26,27)}$. However, other studies have reported no gender difference in $25(\mathrm{OH}) \mathrm{D}$ levels ${ }^{(21,35)}$. Based on our and other studies, we do not believe gender is a useful means of identifying persons at higher risk of vitamin D deficiency.

Strengths of our study include testing of individuals of both genders from ages 18 to 40 years and uniform measurement of serum $25(\mathrm{OH}) \mathrm{D}$ by a single assay. Study weaknesses also exist. The first is the recruitment of relatively few, predominantly Caucasian, study subjects. Aside from milk, we did not query intake of other foods that might contain vitamin $\mathrm{D}$; however, very few other foods contain meaningful doses of vitamin $\mathrm{D}^{(3,36)}$. Additionally, the chemiluminescent assay used for the study may slightly overestimate serum $25(\mathrm{OH}) \mathrm{D}$ compared with the gold standard HPLC assay. We did not record time of day in the sun, although it is known that both season and time of day influence cutaneous vitamin D synthesis ${ }^{(37)}$. We measured 25(OH)D levels in late winter and early spring, a time of low sun exposure in Wisconsin ${ }^{(37)}$. Thus, $25(\mathrm{OH}) \mathrm{D}$ levels were measured at a nadir in our subjects, increasing the likelihood of vitamin D deficiency.

Additional research is needed to refine the current questionnaire and provide cost-effective algorithms to identify individuals who benefit from serum $25(\mathrm{OH}) \mathrm{D}$ measurement. Ideally, a larger study performed in one season would query subjects about sun exposure, milk ingestion and intake of foods (fatty fish, liver, eggs) and supplements containing vitamin D. The study should also assess BMI and smoking, given the higher risk of deficiency reported in obese patients and smokers ${ }^{(21,38,39)}$. Additionally, symptoms or signs of vitamin D deficiency, such as proximal muscle weakness or tibial tenderness, might allow better identification of subjects at high risk of deficiency ${ }^{(40)}$. Ideally, a questionnaire modified from the one herein would provide a 'score' with higher sensitivity and specificity for vitamin D deficiency. Such a tool would prove useful in clinical practice.

Peak bone mass occurs around 30 years of age ${ }^{(41,42)}$. Models indicate that interventions to increase peak bone mass are more effective at preventing osteoporosis than 
interventions later in life ${ }^{(43)}$. Early research suggests that improved vitamin D status promotes peak bone mass ${ }^{(13,14)}$. If researchers confirm the importance of vitamin $\mathrm{D}$ on peak bone mass, young adults would benefit from global vitamin D fortification of food and beverages.

\section{Acknowledgements}

The authors thank the people who participated in this study. We are grateful to Dr Neil Binkley for laboratory assistance with vitamin D assays and Dr Gunnar Thomason for translation of an Icelandic journal article. We thank Andrea N. Jones for editorial assistance. K.E.H. received salary support from NIH grant 1K23 AR050995 during the conduct of this study. All authors were involved in the collection of data and its interpretation, and each author assisted meaningfully in the composition of the manuscript. The individuals cited in the acknowledgement have explicitly agreed to this designation. The authors accept the conditions for submission as explained in the instructions provided. As authors, we have no financial relationship with the sponsor of the study. Sponsors had no role in the design or conduct of the study, nor analysis of the resulting data. If the editors wish to do so, they may review the de-identified study database.

\section{References}

1. Holick MF, Siris ES, Binkley N, Beard MK, Khan A, Katzer JT, Poetruschke RA, Chen E \& dePapp AE (2005) Prevalence of vitamin D inadequacy among postmenopausal North American women receiving osteoporosis therapy. J Clin Endocrinol Metab 90, 3215-3224.

2. Lips P, Hosking DJ, Lippuner K, Norquist JM, Wehren L, Maalouf G, Ragi-Els S \& Chandler J (2006) The prevalence of vitamin D inadequacy amongst women with osteoporosis: an international epidemiological investigation. J Intern Med 260, 245-254.

3. Vieth R (1999) Vitamin D supplementation, 25-hydroxyvitamin D concentrations, and safety. Am J Clin Nutr 69, 842-856.

4. Bischoff-Ferrari HA, Willett WC, Wong JB, Giovannucci E, Dietrich T \& Dawson-Hughes B (2005) Fracture prevention with vitamin D supplementation: a meta-analysis of randomized controlled trials. JAMA 293, 2257-2264.

5. Gartner LM \& Greer FR (2003) Prevention of rickets and vitamin $\mathrm{D}$ deficiency: new guidelines for vitamin $\mathrm{D}$ intake. Pediatrics 111, 908-910.

6. Misra M, Tsai P, Anderson EJ, Hubbard JL, Gallagher K, Soyka LA, Miller KK, Herzog DB \& Klibanski A (2006) Nutrient intake in community-dwelling adolescent girls with anorexia nervosa and in healthy adolescents. $A m J$ Clin Nutr 84, 698-706.

7. Hartman JJ (2000) Vitamin D deficiency rickets in children: prevalence and need for community education. Orthop Nurs 19, 63-67.

8. Cokkinides V, Weinstock M, Glanz K, Albano J, Ward E \& Thun M (2006) Trends in sunburns, sun protection practices, and attitudes toward sun exposure protection and tanning among US adolescents, 1998-2004. Pediatrics 118, 853-864.

9. Schofield PE, Freeman JL, Dixon HG, Borland R \& Hill DJ (2001) Trends in sun protection behaviour among Australian young adults. Aust N Z J Public Health 25, 62-65.
10. Matsuoka LY, Ide L, Wortsman J, MacLaughlin JA \& Holick MF (1987) Sunscreens suppress cutaneous vitamin $\mathrm{D}_{3}$ synthesis. J Clin Endocrinol Metab 64, 1165-1168.

11. Peacey V, Steptoe A, Sanderman R \& Wardle J (2006) Ten-year changes in sun protection behaviors and beliefs of young adults in 13 European countries. Prev Med 43, 460-465.

12. Tucker KL, Morita K, Qiao N, Hannan MT, Cupples LA \& Kiel DP (2006) Colas, but not other carbonated beverages, are associated with low bone mineral density in older women: The Framingham Osteoporosis Study. Am J Clin Nutr 84, 936-942.

13. Valimaki VV, Alfthan H, Lehmuskallio E, Loyttyniemi E, Sahi T, Stenman UH, Suominen H \& Valimaki MJ (2004) Vitamin D status as a determinant of peak bone mass in young Finnish men. J Clin Endocrinol Metab 89, 76-80.

14. Lehtonen-Veromaa MK, Mottonen TT, Nuotio IO, Irjala KM, Leino AE \& Viikari JS (2002) Vitamin D and attainment of peak bone mass among peripubertal Finnish girls: a 3-y prospective study. Am J Clin Nutr 76, 1446-1453.

15. Bischoff-Ferrari HA, Dietrich T, Orav EJ \& Dawson-Hughes B (2004) Positive association between 25-hydroxy vitamin $\mathrm{D}$ levels and bone mineral density: a population-based study of younger and older adults. Am JMed 116, 634-639.

16. Barger-Lux MJ \& Heaney RP (1994) The role of calcium intake in preventing bone fragility, hypertension, and certain cancers. J Nutr 124, 8 Suppl., 1406S-1411S.

17. Garland CF, Garland FC \& Gorham ED (1999) Calcium and vitamin $\mathrm{D}$. Their potential roles in colon and breast cancer prevention. Ann N Y Acad Sci 889, 107-119.

18. Merlino LA, Curtis J, Mikuls TR, Cerhan JR, Criswell LA \& Saag KG (2004) Vitamin D intake is inversely associated with rheumatoid arthritis: results from the Iowa Women's Health Study. Arthritis Rheum 50, 72-77.

19. Tangpricha V, Pearce EN, Chen TC \& Holick MF (2002) Vitamin D insufficiency among free-living healthy young adults. Am J Med 112, 659-662.

20. Sigurdsson G, Franzson L, Porgeirsdottir H \& Steingrimsdottir L (1999) Vitamin D intake and serum 25-OH-vitamin $\mathrm{D}$ concentration in different age groups of Icelandic women. Laeknabladid Med J 85, 398-405.

21. Jacques PF, Felson DT, Tucker KL, Mahnken B, Wilson PW, Rosenberg IH \& Rush D (1997) Plasma 25-hydroxyvitamin $\mathrm{D}$ and its determinants in an elderly population sample. Am J Clin Nutr 66, 929-936.

22. Juttmann JR, Visser TJ, Buurman C, de Kam E \& Birkenhager JC (1981) Seasonal fluctuations in serum concentrations of vitamin D metabolites in normal subjects. Br Med J (Clin Res Ed) 282, 1349-1352.

23. Lensmeyer GL, Wiebe DA, Binkley N, Drezner MK, Singh R \& Darcy TP (2005) Clinically reliable assay for routine monitoring of 25-hydroxyvitamin $\mathrm{D}_{3}$ and 25-hydroxyvitamin $\mathrm{D}_{2}$ in serum using a semi-automated extraction and HPLC. Clin Chem 51, Suppl. A, A192.

24. Binkley N, Krueger D, Cowgill CS, Plum L, Lake E, Hansen KE, DeLuca HF \& Drezner MK (2004) Assay variation confounds the diagnosis of hypovitaminosis D: a call for standardization. J Clin Endocrinol Metab 89, 3152-3157.

25. Ersfeld DL, Rao DS, Body JJ, Sackrison JL Jr, Miller AB, Parikh N, Eskridge TL, Polinske A, Olson GT \& MacFarlane GD (2004) Analytical and clinical validation of the $25 \mathrm{OH}$ vitamin D assay for the LIAISON automated analyzer. Clin Biochem 37, 867-874.

26. Looker AC, Dawson-Hughes B, Calvo MS, Gunter EW \& Sahyoun NR (2002) Serum 25-hydroxyvitamin D status of adolescents and adults in two seasonal subpopulations from NHANES III. Bone 30, 771-777.

27. Zadshir A, Tareen N, Pan D, Norris K \& Martins D (2005) The prevalence of hypovitaminosis D among US adults: data from the NHANES III. Ethn Dis 15, Suppl. 5, S5-97-S5-101. 
28. Dawson-Hughes B, Dallal GE, Krall EA, Harris S, Sokoll LJ \& Falconer G (1991) Effect of vitamin D supplementation on wintertime and overall bone loss in healthy postmenopausal women. Ann Intern Med 115, 505-512.

29. Webb AR \& Holick MF (1988) The role of sunlight in the cutaneous production of vitamin $\mathrm{D}_{3}$. Annu Rev Nutr $\mathbf{8}$, 375-399.

30. Devgun MS, Johnson BE \& Paterson CR (1982) Tanning, protection against sunburn and vitamin $\mathrm{D}$ formation with a UV-A 'sun-bed'. Br J Dermatol 107, 275-284.

31. Tangpricha V, Turner A, Spina C, Decastro S, Chen TC \& Holick MF (2004) Tanning is associated with optimal vitamin D status (serum 25-hydroxyvitamin D concentration) and higher bone mineral density. Am J Clin Nutr 80, $1645-1649$.

32. Salamone LM, Dallal GE, Zantos D, Makrauer F \& DawsonHughes B (1994) Contributions of vitamin D intake and seasonal sunlight exposure to plasma 25-hydroxyvitamin D concentration in elderly women. Am J Clin Nutr 59, 80-86.

33. Sullivan SS, Rosen CJ, Halteman WA, Chen TC \& Holick MF (2005) Adolescent girls in Maine are at risk for vitamin D insufficiency. J Am Diet Assoc 105, 971-974.

34. Haney EM, Stadler D \& Bliziotes MM (2005) Vitamin D insufficiency in internal medicine residents. Calcif Tissue Int 76, 11-16.

35. Rucker D, Allan JA, Fick GH \& Hanley DA (2002) Vitamin D insufficiency in a population of healthy western Canadians. CMAJ 166, 1517-1524.
36. Utiger RD (1998) The need for more vitamin D. $N$ Engl J Med 338, 828-829.

37. Webb AR, Kline L \& Holick MF (1988) Influence of season and latitude on the cutaneous synthesis of vitamin $\mathrm{D}_{3}$ : exposure to winter sunlight in Boston and Edmonton will not promote vitamin $\mathrm{D}_{3}$ synthesis in human skin. $J$ Clin Endocrinol Metab 67, 373-378.

38. Bell NH, Epstein S, Greene A, Shary J, Oexmann MJ \& Shaw S (1985) Evidence for alteration of the vitamin D-endocrine system in obese subjects. J Clin Invest 76, 370-373.

39. Isaia $G$, Giorgino R, Rini GB, Bevilacqua M, Maugeri D \& Adami S (2003) Prevalence of hypovitaminosis D in elderly women in Italy: clinical consequences and risk factors. Osteoporos Int 14, 577-582.

40. Van Veldhuizen PJ, Taylor SA, Williamson S \& Drees BM (2000) Treatment of vitamin D deficiency in patients with metastatic prostate cancer may improve bone pain and muscle strength. J Urol 163, 187-190.

41. Recker RR, Davies KM, Hinders SM, Heaney RP, Stegman MR \& Kimmel DB (1992) Bone gain in young adult women. JAMA 268, 2403-2408.

42. Lin YC, Lyle RM, Weaver CM, McCabe LD, McCabe GP, Johnston CC \& Teegarden D (2003) Peak spine and femoral neck bone mass in young women. Bone 32, 546-553.

43. Hernandez CJ, Beaupre GS \& Carter DR (2003) A theoretical analysis of the relative influences of peak BMD, age-related bone loss and menopause on the development of osteoporosis. Osteoporos Int 14, 843-847. 